\title{
MicroARN et physiopathologie intestinale
}

$>$ Les microARN (miARN) constituent une classe de petits ARN non codants d'environ 20 nucléotides qui contrôlent négativement l'expression d'ARN messagers cibles. Les eucaryotes multicellulaires utilisent les miARN pour contrôler leurs fonctions vitales, différenciation, prolifération ou apoptose. La diversité des miARN et le nombre considérable de leurs ARN messagers cibles en font des acteurs importants de la régulation de l'expression génique. Des études récentes ont révélé que l'expression anormale des miARN représente une caractéristique commune des cellules cancéreuses et que ces miARN peuvent fonctionner comme des gènes suppresseurs de tumeur ou des oncogènes. Cette revue fait le point des travaux portant sur l'implication des miARN dans la pathogénie des cancers colorectaux. Le rôle des miARN dans le contrôle de l'inflammation et leur implication potentielle dans les pathologies inflammatoires intestinales sont aussi discutés. <
En 1993, Ambros et al. ont rapporté que lin-4, un gène de $C$. elegans, ne codait pas pour une protéine mais pour un ARN d'environ 20 nucléotides dont l'invalidation affectait le développement du ver [1]. D'abord perçus comme une «bizarrerie » propre à C. elegans, des petits ARN sont maintenant décrits dans tout le phylum. De façon remarquable, certains d'entre eux sont très conservés au cours de l'évolution. Les membres de cette nouvelle classe d'ARN ont été collectivement appelés microARN ou miARN. On estime actuellement qu'il en existe au moins 500 chez l'homme (pour revue, voir [2-5]). Les miARN sont majoritairement issus de transcrits produits par I'ARN polymérase II (Pol II) : il peut s'agir de transcrits autonomes (l'information génétique étant celle du miARN) ou de transcrits mixtes (comprenant une région codante et un miARN contenu dans un intron ou dans une région 3' non codante). Cependant, une étude récente démontre que l'expression de certains miARN requiert I'ARN polymérase III, suggérant que

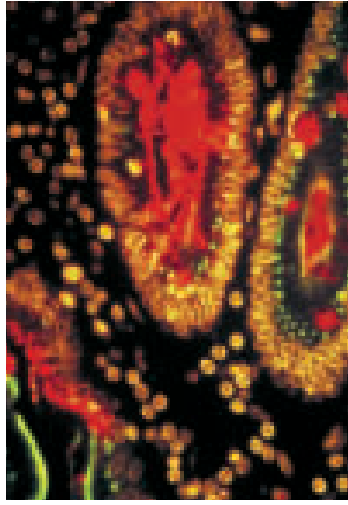

Inserm, U773, Centre

de Recherche Bichat Beaujon

CRB3, BP 416,

F-75870, Paris, France.

Université Paris 7 Denis Diderot,

site Bichat, BP 416,

F-75018, Paris, France.

Eric.0gier-Denis@bichat.inserm.fr

la connexion entre les miARN et les éléments transposables (séquences Alu) est plus importante qu'initialement admise [6].

Les miARN sont synthétisés sous forme d'ARN précurseurs de grande taille dont la maturation fait intervenir deux RNases III, Drosha et Dicer (Figure 1) [7-10]. En conjonction avec son partenaire DGCR8 [11, 12], Drosha convertit, dans le noyau, un transcrit primaire (pri-miARN) en une structure en épingle à cheveux irrégulière d'environ 70 nucléotides (pré-miARN). Une fois transféré dans le cytoplasme par l'exportine 5, le pré-miARN est pris en charge par Dicer qui façonne un intermédiaire de maturation dont un seul des deux brins formera le miARN mature (Figure 1) $[13,14]$. Au cours de ce processus de maturation, les miARN interagissent avec des protéines spécifiques pour former un complexe ribonucléoprotéique stable dénommé RISC-miRNP (RNA-induced silencing complex-micro-ribonucleoprotein). Au sein de ce complexe, le miARN interagit avec des séquences complémentaires de la partie 3' UTR des ARNm cibles. Les modalités d'action de RISC-miRNP sont encore mal comprises, mais semblent dépendre de la complémentarité du duplex miARN:ARNm mise en jeu et de la nature des protéines adaptatrices Argonautes qui le composent. Si cette complémentarité est totale, RISC-miRNP induit alors un clivage endonucléolytique au milieu de l'hybride miARN:ARNm entraînant la dégradation rapide de l'ARNm (Figure I). En revanche, si cette complémentarité est partielle, l'inhibition de la traduction de I'ARNm cible est favorisée et la stabilité de la cible peut 


\begin{tabular}{|c|c|c|c|c|}
\hline miARN & Localisation & Cancers & Fonction & Références \\
\hline $\begin{array}{l}\operatorname{miR}-15 a \\
\operatorname{miR}-16-1\end{array}$ & $\begin{array}{l}\text { Chromosome } \\
13 q 14\end{array}$ & Leucémies lymphoïdes & $\begin{array}{l}\text { Suppresseur de tumeur régule négativement } \\
\mathrm{Bcl}-2\end{array}$ & {$[55,56]$} \\
\hline $\begin{array}{l}\text { miR-143 } \\
\text { miR-145 }\end{array}$ & $\begin{array}{l}\text { Chromosome } \\
5 q 32-33\end{array}$ & $\begin{array}{l}\text { Colorectal, sein, prostate, } \\
\text { Leucémie, lymphoïde }\end{array}$ & $\begin{array}{l}\text { Suppresseur de tumeur cibles non encore iden- } \\
\text { tifiées }\end{array}$ & {$[57,58]$} \\
\hline $\operatorname{miR}-21$ & $\begin{array}{l}\text { Chromosome } \\
17 q 23.2\end{array}$ & $\begin{array}{l}\text { Glioblastome, sein } \\
\text { Cholangiocarcinome }\end{array}$ & Oncogène régule négativement l'apoptose & {$[57,59]$} \\
\hline Famille Let7 & Locus multiples & Colorectal, poumon & $\begin{array}{l}\text { Suppresseur de tumeur régule négativement Ras } \\
\text { et c-myc }\end{array}$ & {$[60-62]$} \\
\hline miR-142 & $\begin{array}{l}\text { Chromosome } \\
17 q 22\end{array}$ & Leucémies & Oncogène régule positivement c-myc & [63] \\
\hline miR-155 & $\begin{array}{l}\text { Chromosome } \\
2 \text { lq21 }\end{array}$ & $\begin{array}{l}\text { Lymphome de Burkitt, Hodgkin, } \\
\text { Cancer du sein et gastrique }\end{array}$ & $\begin{array}{l}\text { Oncogène régule négativement } \\
\text { Tyrosine phosphatase PLR-3 }\end{array}$ & {$[57,64,65]$} \\
\hline miR-17-92 & $\begin{array}{l}\text { Chromosome } \\
13 q 31-3\end{array}$ & $\begin{array}{l}\text { Carcinome hépatocellulaire, } \\
\text { lymphomes B, cancer du poumon }\end{array}$ & Oncogène cibles non encore identifiées & {$[66-68]$} \\
\hline $\begin{array}{l}\text { miR-31 } \\
\text { miR-96 } \\
\text { miR-133b } \\
\text { miR-135b }\end{array}$ & Locus multiples & Colorectal & Oncogènes cibles non encore identifiées & [69] \\
\hline
\end{tabular}

Tableau I. MicroARN associés aux cancers humains.

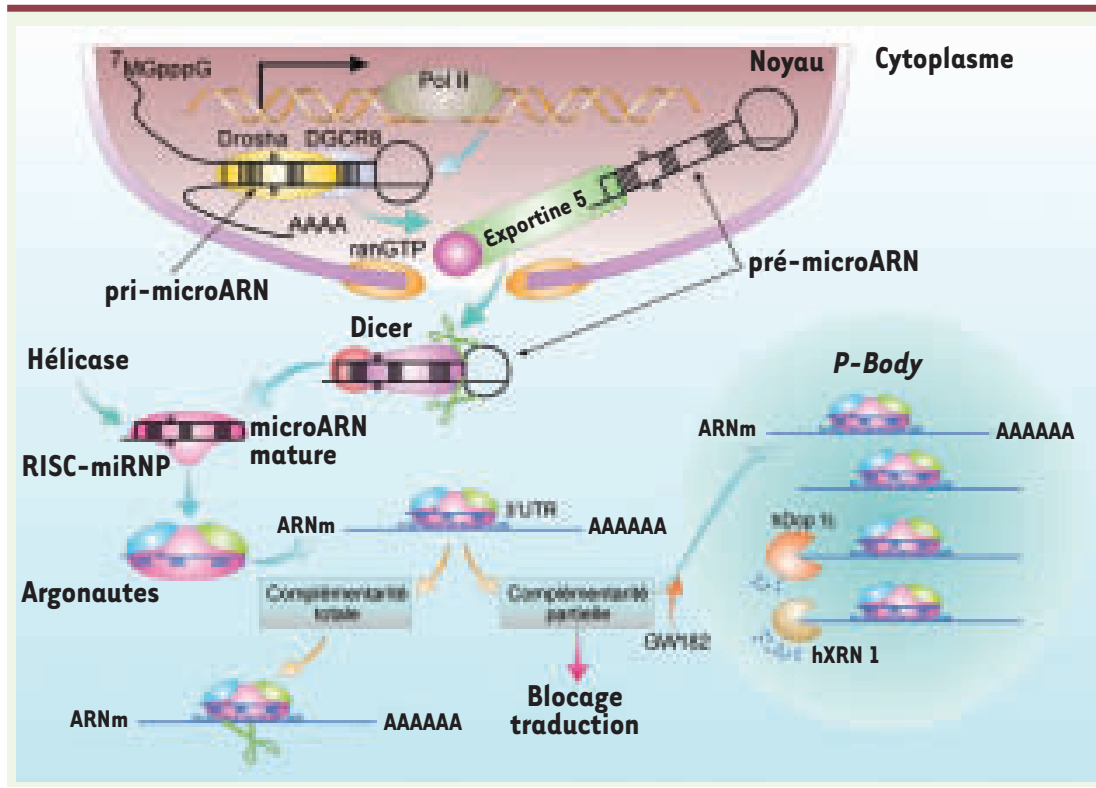

Figure 1. Biogenèse des microARN. Les gènes des microARN (miARN) sont transcrits par une ARN polymérase II ( Pol II) dans le noyau pour former des intermédiaires de biosynthèse de grande taille, les pri-miARN (primary miRNA), qui sont majoritairement cappés ( $\left.{ }^{7} \mathrm{MGpppG}\right)$ et polyadénylés (AAAA) et contiennent une ou plusieurs structures en épingle à cheveux. Les pri-miARN sont ensuite modifiés par une RNase III, Drosha en association avec son co-facteur DGCR8 (DiGeorge syndrome critical region gene 8 ou Pasha chez la drosophile), pour former des produits d'environ 70 nucléotides, les prémiARN (precursor miRNA). L'exportine 5 et Ran-GTP permettent la translocation du prémiARN dans le cytosol. Celui-ci est clivé par une seconde RNase III, Dicer, engendrant un duplex d'environ 20 nucléotides. Le miARN mature est alors transféré au complexe multiprotéique

RISC-miRNP (RNA-induced silencing complex-micro-ribonucleoprotein). Une hélicase facilite la séparation du double brin du duplex et l'association de protéines Argonautes stabilise le brin monocaténaire du miARN au sein de RISC-miRNP. Le miARN mature contrôle négativement l'expression protéique et la stabilité de sa cible en se fixant sur une séquence complémentaire de la partie 3'-UTR de l'ARNm cible au niveau des polysomes. Le degré de complémentarité détermine le devenir de l'ARNm. Si cette complémentarité est totale, le miARN entraîne la dégradation de l'ARNm (coupure endonucléolytique) par Argonaute 2, alors qu'une complémentarité partielle inhibe l'initiation et/ou l'élongation de la traduction et peut aboutir à la dégradation exonucléolytique de l'ARNm dans des compartiments cytosoliques dynamiques, les $P$-bodies ou GW-bodies. Ces structures contiennent une forte concentration de molécules impliquées dans la dégradation des ARNm (désadénylase, Decapping complex hDCPl/2 et 5'-3' exonucléase hXRN1). Le ciblage des duplexes miARN/ARNm dans les $P$-bodies ferait intervenir un composant structural des $P$-bodies, la protéine GW182. 
être affectée par un mécanisme de dégradation différent du clivage endonucléolytique [15, 16] (Figure 1), le raccourcissement de la queue poly(A) étant l'étape initiale de cette dégradation [17, 18]. Ces évènements posttranscriptionnels auraient lieu dans des structures cytoplasmiques dynamiques, appelées $P$-bodies ou GW bodies, vraisemblablement en réponse à la répression de la traduction [19-21]. Les $P$-bodies contiennent une forte concentration de molécules impliquées dans la dégradation des ARNm, comme la déadénylase, le complexe de decapping Dcpl/2 et la 5'-3' exonucléase XRN1 (revue dans [22]) (Figure 1). Plusieurs études démontrent que la formation des $P$-bodies peut être dépendante de l'expression de miARN, notamment celle du miARN let-7 [23-25], et que leur déstabilisation, par ARN interférence contre l'un de leurs constituants (Lsml), n'affecte pas la répression de la traduction induite par les miARN [21]. Cependant, si les $P$-bodies sont importants pour la voie des miARN, ils ne peuvent être restreints à cette seule activité puisqu'ils ont été initialement décrits chez $S$. cerevisiae, un organisme qui ne contient aucun des gènes de la machinerie de l'interférence ARN.

\section{Fonctions des microARN}

Il est maintenant bien établi que les miARN contrôlent de nombreux processus physiologiques, par exemple le développement, la différenciation et la prolifération cellulaires, l'apoptose ou la réponse au stress. Des mutations des miARN let- 7 et lin-4 observées chez $C$. elegans affectent le contrôle du développement du ver [1]. La perte de fonction de lin-4 induit une différenciation anormale de lignages cellulaires spécifiques et la réitération des types cellulaires embryonnaires aux stades tardifs du développement du nématode. La famille let-7 est également impliquée dans la régulation du proto-oncogène Ras, et les miARN bantam et miR-14 sont des régulateurs essentiels de la mort cellulaire programmée chez D. melanogaster [26]. L'inhibition de l'expression de la famille miR2/6/11/13/308 induit l'apoptose en contrôlant les facteurs proapoptotiques hid, grim, reaper et sickle dans les embryons de drosophiles [27]. Certains miARN ont des fonctions essentielles dans le développement et contrôlent la différenciation cellulaire dans divers tissus. Par exemple, miR-273 et lys-6 sont impliqués dans la différenciation neuronale de $C$. elegans [28], miR-181 dans la différenciation des cellules hématopoïétiques humaines [29], miR-375 dans le développement des îlots pancréatiques de mammifères [30] et dans la régulation de la sécrétion de l'insuline, miR-143 dans la différenciation adipocytaire [31] et miR-1 dans le développement cardiaque humain [32]. De plus, les miARN pourraient être impliqués dans la surveillance des profils d'expression génique et le filtrage des bruits transcriptionnels assurant l'identité tissulaire et, de ce fait, seraient les garants de l'exactitude des programmes d'expression au cours du développement [33].

Bien que leur nombre ne cesse de croître et que leurs fonctions régulatrices se diversifient, le rôle des miARN dans les pathologies humaines est encore mal compris. Un lien direct entre la fonction des miARN et la cancérogenèse a été démontré récemment. Plus de la moitié des miARN identifiés à ce jour sont localisés dans des régions génomiques qui sont fréquemment amplifiées, délétées ou réarrangées dans les cancers humains suggérant que les anomalies d'expression des miARN pourraient jouer un rôle dans la pathogenèse des cancers [34]. Un niveau de complexité supplémentaire vient de ce que la maturation des précurseurs des miARN est également contrôlée au niveau post-transcriptionnel. Ainsi, un défaut d'activité de Drosha pourrait expliquer la production diminuée de miARN matures observée couramment dans les cellules tumorales [35].

Le dérèglement de l'expression des miARN a été directement observé dans divers cancers : lymphome de Burkitt [36], cancer du sein [37], cancer du poumon [38], glioblastome [39] ou cancer colorectal [40] (Tableau I). Les liens entre altérations des miARN et cancérogenèse commencent à être compris. He et al. [41] ont montré qu'un groupe de 7 miARN (cluster polycistronique miR17-92) est associé au développement de lymphomes de type B. Dans un modèle de lymphome de souris, l'expression forcée de ce cluster augmente l'expression de l'oncogène c-Myc, l'incidence du cancer et la rapidité de son développement. Chez l'homme, Calin et al. [42] ont montré récemment que l'expression de 13 miARN dans les lymphomes, incluant miR-15a et miR-16-1, constitue une signature de la progression de la maladie. Certains résultats de la littérature apparaissent cependant contradictoires. En effet, O'Donnell et al. [43] ont montré qu'un ensemble de six miARN (miR-17-5p, miR-18, miR-19, miR-20, miR-92 et miR-106) pourrait freiner l'apparition des lymphomes en contrôlant l'effet d'emballement induit par deux gènes, l'oncogène $c-M y c$ et un facteur de transcription $\varepsilon 2 F 1$, qui se stimulent l'un l'autre. Ces données sont autant d'arguments démontrant le rôle joué par certains miRNA dans les cancers. Par ailleurs, Lu et al. [44] ont montré que les mesures d'expression de 217 miARN, réalisées par cytométrie en flux sur 330 échantillons de cellules de patients atteints de différentes leucémies, représentaient un meilleur indicateur du lignage en cause et de l'état de différenciation des cellules leucémiques que les données de 16000 ARN messagers, en accord avec un rôle déterminant des miARN dans le contrôle de la différenciation.

\section{Émergence des microARN en physiopathologie intestinale}

Les pathologies intestinales humaines, cancer colorectal et maladies inflammatoires chroniques intestinales ( $\mathrm{MICl}$ ) (maladie de Crohn et rectocolite hémorragique), sont la résultante d'interactions complexes entre mutations/polymorphismes génétiques et facteurs environnementaux. Malgré une meilleure compréhension des voies de signalisation et l'identification de mutations fonctionnelles de certains gènes, les mécanismes moléculaires gouvernant l'expression d'un grand nombre de 
protéines clés dont le dérèglement intervient dans la pathogénie des ces maladies intestinales restent largement inconnus. Une question récurrente dans la compréhension de l'étiologie ces maladies est de savoir si un mécanisme moléculaire commun peut être à l'origine d'un dérèglement aussi important. Les miARN, par leur diversité d'action sur l'expression de nombreux messagers, pourraient représenter le chaînon manquant dans la compréhension des pathologies intestinales.

\section{MicroARN et cancer colorectaux}

L'implication des miARN dans la cancérogenèse colorectale a été initialement démontrée par Michael et al. [40] et confirmée depuis [45]. Vingt-huit miARN sont sous- ou sur-exprimés dans des biopsies d'adénocarcinomes coliques par rapport à leur expression dans la muqueuse colique normale. En particulier, l'expression de miR-143 et miR-145 est fortement atténuée. L'analyse in silico des ARNm susceptibles d'être modulés par ces deux miARN identifie plusieurs composants impliqués dans la régulation des ARNm et des protéines ( $\varepsilon R$ protein $p 44$, thiorédoxine), de voies transductionnelles (Raf, Rho) et de processus métaboliques (dihydroxyfolate réductase). Parallèlement, Akao et al. [46] ont montré une expression réduite de let-7 dans les tumeurs colorectales et dans des lignées d'adénocarcinome colique. La transfection de let7 dans ces lignées induit une diminution de leur croissance associée à une inhibition d'expression des oncogènes Ras et $c$-myc démontrant que let- 7 se comporte comme un gène suppresseur de tumeur. Plus récemment, Bandrés et al. [47] ont examiné l'expression de 156 miARN dans des cancers colorectaux et dans 16 lignées cellulaires intestinales cancéreuses. L'expression de 13 de ces miARN est altérée dans les tumeurs et dans les lignées comparativement à des échantillons de tissus non tumoraux. L'expression de 5 miARN de ce groupe est également altérée dans d'autres types de cancers, dont les cancers du sein, du poumon, les glioblastomes et les leucémies. Parmi les miARN dont l'expression dans les tumeurs et les lignées colorectales diffère de ce qu'elle est dans la muqueuse normale, quatre (miR-31, miR-96, miR$133 b$ et miR-135b) sont sur-exprimés et deux (miR-145 et miR-183) sous-exprimés, suggérant qu'ils se comportent respectivement comme des oncogènes ou des gènes suppresseurs de tumeur. Enfin, des analyses de séquences à grande échelle révèlent que plus de $46 \%$ des promoteurs putatifs contrôlant l'expression des 326 miARN humains connus à ce jour, sont des cibles de la protéine $\mathrm{p} 53$. L'invalidation de $\mathrm{p} 53$ par ARN interférence dans des cellules HCT-116 dérivées d'adénocarcinome colique module l'expression de près de 200 ARNm qui sont soumis à une régulation post-transcriptionnelle et sont tous des cibles potentielles de miARN. Ces résultats suggèrent que $p 53$ et certains miARN influencent, de façon coordonnée, l'expression de multiples gènes via un mécanisme de régulation intégrée [48]. Tout récemment, une approche d'analyse miRAGE (miRNA serial analysis of gene expression) [49], combinant les approches de clonage des miARN et d'analyse d'expression génique, a permis d'identifier dans des cellules de cancer colorectal 200 miARN connus et 133 nouveaux miARN candidats, démontrant que le catalogue des miARN ne cesse de s'enrichir. Dans ce contexte, la connaissance des modifications d'expression des miARN qui ciblent spécifiquement différents gènes impliqués dans la pathogenèse des cancers colorectaux devrait permettre de mieux comprendre les mécanismes moléculaires intimes qui gouvernent la cancérogenèse colorectale.

\section{MicroARN et maladies inflammatoires chroniques de l'intestin ( $\mathrm{MICl}$ )}

Les $\mathrm{MICl}$ regroupent des affections inflammatoires comme la maladie de Crohn ou la rectocolite hémorragique, touchant le tube digestif, en particulier l'intestin et le côlon. Si l'étiologie des $\mathrm{MICl}$ reste inconnue, l'importance de facteurs génétiques, infectieux et immunologiques semble prédominante. Ce terrain complexe et multifactoriel devrait représenter une cible de choix pour les études des miARN, mais rien n'est encore publié à ce jour. Pourtant, le rôle des miARN dans le processus inflammation commence à émerger dans la littérature. L'une des premières études a porté sur le contrôle post-transcriptionnel et la stabilité des ARNm dans la régulation de l'expression de gènes pro-inflammatoires, en mettant l'accent sur les séquences ARE (AU-rich elements) identifiées dans la partie 3'-UTR de plusieurs cytokines. Ces séquences ARE, qui agissent en cis, sont des éléments déstabilisants et activent le turn-over rapide des messagers. Or Dicer et Argonaute, les composants clés de la machinerie miARN/ARN interférence, sont impliqués dans l'instabilité des ARNm guidée par les séquences ARE dans des cellules de drosophile et des cellules Hela [50]. Des analyses de séquences révèlent que des miARN (miR-16, miR-289, miR-227 et miR-304) comportent une séquence complémentaire de celle des séquences ARE et augmentent l'instabilité des ARNm spécifiant des cytokines pro-inflammatoires: le TNF- $\alpha$, le GM-CSF (granulocyte-macrophage colony-stimulating factor) et les interleukines (IL)-6 et -8. Il est donc probable que les miARN exercent une fonction régulatrice majeure dans l'expression de certains médiateurs de l'inflammation. Récemment, Navarro et al. [51] ont montré que, chez la plante Arabidopsis, les miARN participent à l'établissement de la résistance bactérienne et à la régulation de la défense de l'hôte. Ainsi, la flagelline, un dérivé peptidique bactérien, induit un changement rapide du taux de transcrits chez Arabidopsis via la surexpression du miARN miR-393. L'analyse des cibles potentielles de miR-393, dont l'expression est modifiée par la flagelline, identifie quatre récepteurs à l'auxine (TIRl et AFBl-3), une hormone impliquée dans l'élongation de la plante et qui exerce un fort pouvoir rhyzogène. De façon tout à fait originale, ces auteurs ont démontré que la répression de la signalisation induite par l'auxine active la réponse immunitaire générale de la plante et favorise sa résistance à l'infection par une souche bactérienne virulente. Par ailleurs, l'analyse du profil d'expression de 200 miARN dans des monocytes humains a permis de montrer que plusieurs miARN peuvent être consi- 
dérés comme des gènes de réponse aux endotoxines bactériennes [52]. Une grande variété de composés bactériens, comme le lypopolysaccharide (LPS) des bactéries Gram-négatif, et des cytokines pro-inflammatoires, induisent l'expression de miR-146a et miR-146b dans les cellules THP-1 (une lignée monocytaire) de manière dépendante de NF-KB. L'analyse prédictive des cibles de miR-146a/b a permis d'identifier deux adaptateurs clés de la chaîne transductionnelle des récepteurs TLR (Toll-like receptors) et cytokiniques, TRAF6 (TNF receptor-associated factor 6 ) et IRAKI (IL-1 receptor-associated kinase 1 ). Ainsi, miR-146a/b pourraient intervenir dans la régulation négative de la signalisation des TLR et des cytokines en réprimant l'expression de ces adaptateurs participant ainsi à l'extinction de la réponse inflammatoire.

La caractérisation des miARN exprimés ou réprimés dans les $\mathrm{MICl}$ et l'identification de leurs cibles, en particulier celles qui gouvernent l'équilibre de la réponse aux récepteurs TLR et cytokiniques, devraient permettre rapidement de mieux comprendre l'étiologie de ces maladies complexes et éventuellement de développer de nouvelles thérapeutiques même si de nombreux écueils sur le plan pharmacologique (biodisponibilité, toxicité et spécificité) persistent.

\section{Conclusions}

Nous sommes à l'aube de la compréhension du rôle des miARN dans les pathologies intestinales humaines. Identifier dans ces pathologies le répertoire complet des miARN et de leurs ARNm cibles représente un réel défi, à la fois expérimental et conceptuel. Même si certains ARNm cibles ont été récemment prédits par des approches in silico [53], il conviendra de développer rapidement des approches biochimiques et génétiques pour valider formellement ces interactions. Appréhender la combinatoire d'interactions ARN:miARN et les réseaux de régulation sous-jacents ajoute un autre niveau de complexité qu'il faudra décrypter pour apprécier pleinement la fonction biologique de cette nouvelle famille d'ARN non codants. Des phénomènes épigénétiques de «mise en veille» de gènes faisant intervenir des mécanismes apparentés à l'ARN interférence $(\mathrm{ARNi})$ ont été récemment décrits, notamment chez les plantes et S. pombe [54]. L'intervention de miARN dans de tels processus chez les vertébrés reste une question ouverte qu'il conviendra aussi d'éclaircir. II est d'ores et déjà acquis que la découverte des miARN, et plus généralement celle de la machinerie de l'ARNi, a révolutionné notre vision du contrôle de l'expression des gènes. Les miARN apparaissent comme des acteurs importants de la cancérogenèse colique. Nul doute qu'ils jouent également un rôle majeur dans les pathologies inflammatoires intestinales. Ils représentent donc un réel enjeu en recherche fondamentale pour comprendre leur(s) mécanisme(s) d'action et en santé publique pour améliorer les traitements en vigueur. $\diamond$

\section{SUMMARY}

MicroRNAs and intestinal pathophysiology

MicroRNAs (miRNAs) represent an abundant class of endogenously expressed small RNAs, which is believed to control the expression of proteins through specific interaction with their mRNAs. MiRNAs are non-coding RNAs of 18 to 24 nucleotides that negatively regulate target mRNAs by binding to their 3'-untranslated regions (UTR). Most eukaryotic cells utilize miRNA to regulate vital functions such as cell differentiation, proliferation or apopotosis. The diversity of miRNAs and of their mRNA targets strongly indicate that they play a key role in the regulation of protein expression. To date, more than 500 different miRNAs have been identified in animals and plants. There are at least 326 miRNAs in the human genome, comprising 1-4\% of all expressed human genes, which makes miRNAs one of the largest classes of gene regulators. A single miRNA can bind to and regulate many different mRNA targets and, conversely, several different miRNAs can bind to and cooperatively control a single mRNA target. The correlation between the expression of miRNAs and their effects on tumorigenesis and on the proliferation of cancer cells is beginning to gain experimental evidences. Recent studies showed that abnormal expression of miRNAs represents a common feature of cancer cells and that they can function as tumor suppressor genes or as oncogenes. Therefore, this diversity of action for miRNAs on several target genes could be one of the common mechanisms involved in the deregulation of protein expression observed during intestinal disorders. In this review, the emergent functions of miRNAs in colorectal cancer and their potential role in the intestinal inflammatory process are discussed. $\diamond$

\section{REMERCIEMENTS}

Nous tenons à remercier chaleureusement M. Jean-Pierre Laigneau (Inserm U773) pour la réalisation des illustrations de cette synthèse ainsi que le Dr Daniel Maréchal (New Product \& Business Development Manager) et le Dr. Marie-Claire Beckers (Product Manager) de la société Eurogentec (Seraing, Belgique) pour leur collaboration scientifique. Nous remercions les différents organismes publics ou associatifs pour leur financement: l'Institut National de la Santé et de la Recherche Médicale (Inserm), I'Université Paris VII, le Centre National de la Recherche Scientifique (CNRS), l'Association François Aupetit AFA (Eric Ogier-Denis) et la Ligue Nationale contre le Cancer (Eric Ogier-Denis, financement projet $n^{\circ}$ R05/75-103 2005-2006).

\section{RÉFÉRENCES}

1. Lee RC, Feinbaum RL and Ambros V. The C. elegans heterochronic gene lin-4 encodes small RNAs with antisense complementarity to lin-14. Cell 1993; $75: 843-54$.

2. Lewis BP, Burge CB, Bartel DP. Conserved seed pairing, often flanked by adenosines, indicates that thousands of human genes are microRNA targets. Cell $2005 ; 120: 15-20$.

3. Xie X, Lu J, Kulbokas $\varepsilon$ J, et al. Systematic discovery of regulatory motifs in human promoters and 3' UTRs by comparison of several mammals. Nature $2005 ; 434: 338-45$

4. Bentwich I, Avniel A, Karov Y, et al. Identification of hundreds of conserved and nonconserved human microRNAs. Nat Genet 2005 ; 37 : 766-70.

5. Calin GA, Croce CM. MicroRNA signatures in human cancers. Nat Rev Cancer $2006 ; 6: 857-66$

6. Borchert GM, Lanier W, Davidson BL. RNA polymerase III transcribes human microRNAs. Nat Struct Mol Biol 2006 ; 13 : 1097-101.

7. Hutvagner G, McLachlan J, Pasquinelli AE, et al. A cellular function for the RNA-interference enzyme Dicer in the maturation of the let-7 small temporal RNA. Science $2001 ; 293: 834-8$. 
8. Lee Y, Ahn C, Han J, et al. The nuclear RNase III Drosha initiates microRNA processing. Nature $2003 ; 425: 415-9$

9. Lee YS, Nakahara K, Pham JW, et al. Distinct roles for Drosophila Dicer-1 and Dicer-2 in the siRNA/miRNA silencing pathways. Cell $2004 ; 117: 69-81$.

10. Cavaillé J. Des microARN comme s'il en pleuvait... Med Sci (Paris) $2004 ; 20: 399-401$

11. Han J, Lee Y, Yeom KH, et al. The Drosha-DGCR8 complex in primary microRNA processing. Genes Dev $2004 ; 18$ : 3016-27.

12. Yeom KH, Lee Y, Han J, Suh MR, Kim VN. Characterization of DGCR8/Pasha, the essential cofactor for Drosha in primary miRNA processing. Nucleic Acids Res 2006 ; 34 : 4622-9.

13. Khvorova A, Reynolds A, Jayasena, SD. Functional siRNAs and miRNAs exhibit strand bias. Cell $2003 ; 115: 209-16$

14. Schwarz DS, Hutvagner G, Du T, et al. Asymmetry in the assembly of the RNAi enzyme complex. Cell $2003 ; 115: 199-208$.

15. Lim LP, Lau NC, Garrett-Engele P, et al. Microarray analysis shows that some microRNAs downregulate large numbers of target mRNAs. Nature $2005 ; 433: 769-73$.

16. Bagga $S$, Bracht J, Hunter $S$, et al. Regulation by let- 7 and lin-4 miRNAs results in target mRNA degradation. Cell $2005 ; 122: 553-63$.

17. Giraldez AJ, Mishima Y, Rihel J, et al. Zebrafish MiR-430 promotes deadenylation and clearance of maternal mRNAs. Science 2006 ; $312: 75-9$.

18. Wu L, Fan J, Belasco JG. MicroRNAs direct rapid deadenylation of mRNA. Proc Natl Acad Sci USA $2006 ; 103: 4034-9$

19. Liu J, Valencia-Sanchez MA, Hannon GJ, Parker R. MicroRNA-dependent localization of targeted mRNAs to mammalian P-bodies. Nat Cell Biol $2005 ; 7: 719-23$.

20. Engels BM, Hutvagner $G$. Principles and effects of microRNA-mediated post-transcriptional gene regulation. Oncogene $2006 ; 25: 6163-9$.

21. Chu CY, Rana TM. Translation repression in human cells by microRNA-induced gene silencing requires RCK/p54. PLoS Biol 2006; 4 : e210.

22. Newbury SF, Muhlemann 0 , Stoecklin, G. Turnover in the Alps: an mRNA perspective. Workshops on mechanisms and regulation of mRNA turnover. EMBO Rep 2006; 7: 143-8.

23. Pauley KM, Eystathioy T, Jakymiw A, et al. Formation of GW bodies is a consequence of microRNA genesis. EMBO Rep 2006 ; 7 : 904-10.

24. Rehwinkel J, Behm-Ansmant I, Gatfield D, Izaurralde $\varepsilon$. A crucial role for GW182 and the DCP1: DCP2 decapping complex in miRNA-mediated gene silencing. RNA 2005; $11: 1640-7$.

25. Bruno I, Wilkinson MF. P-bodies react to stress and nonsense. Cell $2006 ; 125: 1036-8$

26. Brennecke J, Hipfner DR, Stark A, et al. bantam encodes a developmentally regulated microRNA that controls cell proliferation and regulates the proapoptotic gene hid in Drosophila. Cell 2003; 113: 25-36.

27. Leaman D, Chen Py, Fak J, et al. Antisense-mediated depletion reveals essential and specific functions of microRNAs in Drosophila development. Cell $2005 ; 121: 1097-108$.

28. Chang S, Johnston RJ Jr, Frokjaer-Jensen C, et al. MicroRNAs act sequentially and asymmetrically to control chemosensory laterality in the nematode. Nature $2004 ; 430: 785-9$.

29. Chen CZ, Li L, Lodish HF, Bartel DP. MicroRNAs modulate hematopoietic lineage differentiation. Science 2004 ; 303: 83-6.

30. Poy MN, Eliasson L, Krutzfeldt J, et al. A pancreatic islet-specific microRNA regulates insulin secretion. Nature $2004 ; 432: 226-30$.

31. Esau C, Kang X, Peralta $\varepsilon$, et al. MicroRNA-143 regulates adipocyte differentiation. J Biol Chem $2004 ; 279: 52361-5$

32. Zhao $Y$, Samal $\varepsilon$, Srivastava D. Serum response factor regulates a muscle-specific microRNA that targets Hand2 during cardiogenesis. Nature 2005 ; 436 : 214-20.

33. Stark A, Brennecke J, Bushati N, et al. Animal MicroRNAs confer robustness to gene expression and have a significant impact on 3'UTR evolution. Cell $2005 ; 123$ : 1133-46.

34. Calin GA, Sevignani C, Dumitru CD, et al. Human microRNA genes are frequently located at fragile sites and genomic regions involved in cancers. Proc Natl Acad Sci USA 2004 . $101: 2999-3004$.

35. Thomson JM, Newman M, Parker JS, et al. Extensive post-transcriptional regulation of microRNAs and its implications for cancer. Genes Dev 2006; $20: 2202-7$.

36. Metzler M, Wilda M, Busch K, et al. High expression of precursor microRNA-155/BIC RNA in children with Burkitt lymphoma. Genes Chrom Cancer 2004 ; 39 : 167-9.

37. Iorio MV, Ferracin M, Liu CG, et al. MicroRNA gene expression deregulation in human breast cancer. Cancer Res $2005 ; 65: 7065-70$.

38. Takamizawa J, Konishi H, Yanagisawa K, et al. Reduced expression of the let-7 microRNAs in human lung cancers in association with shortened postoperative survival. Cancer Res 2004 ; $64: 3753-6$.

39. Chan JA, Krichevsky AM, Kosik KS. MicroRNA-21 is an antiapoptotic factor in human glioblastoma cells. Cancer Res $2005 ; 65: 6029$-33.

40. Michael MZ, SM OC, van Holst Pellekaan NG, Young, GP, James RJ. Reduced accumulation of specific microRNAs in colorectal neoplasia. Mol Cancer Res 2003; $1: 882-91$.

41. He L, Thomson JM, Hemann MT, et al. microRNA polycistron as a potential human oncogene. Nature $2005 ; 435: 828-33$.

42. Calin GA, Ferracin M, Cimmino A, et al. A MicroRNA signature associated with prognosis and progression in chronic lymphocytic leukemia. $N$ Engl J Med 2005 ; 353 : 1793-801.

43. O'Donnell KA, Wentzel $\varepsilon A$, Zeller Kl, et al. c-Myc-regulated microRNAs modulate $\varepsilon 2 \mathrm{Fl}$ expression. Nature $2005 ; 435: 839-43$
44. Lu J, Getz G, Miska EA, et al. MicroRNA expression profiles classify human cancers. Nature $2005 ; 435: 834-8$

45. Volinia $S$, Calin GA, Liu CG, et al. A microRNA expression signature of human solid tumors defines cancer gene targets. Proc Natl Acad Sci USA $2006 ; 103: 2257-61$.

46. Akao $Y$, Nakagawa $Y$, Naoe T. let-7 microRNA functions as a potential growth suppressor in human colon cancer cells. Biol Pharm Bull 2006 ; 29 : $903-6$.

47. Bandres $\varepsilon$, Cubedo $\varepsilon$, Agirre $X$, et al. Identification by Real-time PCR of 13 mature microRNAs differentially expressed in colorectal cancer and non-tumoral tissues. Mol Cancer $2006 ; 5: 29$.

48. Xi Y, Shalgi R, Fodstad 0 , Pilpel Y, Ju J. Differentially regulated microRNAs and actively translated messenger RNA transcripts by tumor suppressor p53 in colon cancer. Clin Cancer Res 2006 ; 12 : 2014-24.

49. Cummins JM, He Y, Leary RJ, et al. The colorectal microRNAome. Proc Natl Acad Sci USA $2006 ; 103: 3687-92$.

50. Jing $Q$, Huang $S$, Guth $S$, et al. Involvement of microRNA in AU-rich element-mediated mRNA instability. Cell 2005 ; 120 : 623-34.

51. Navarro L, Dunoyer $P$, Jay F, et al. A plant miRNA contributes to antibacterial resistance by repressing auxin signaling. Science 2006 ; $312: 436-9$

52. Taganov KD, Boldin MP, Chang KJ, Baltimore D. NF-kappaB-dependent induction of microRNA miR-146, an inhibitor targeted to signaling proteins of innate immune responses. Proc Natl Acad Sci USA 2006 $103: 12481-6$

53. Lewis BP, Shih IH, Jones-Rhoades MW, Bartel DP, Burge CB. Prediction of mammalian microRNA targets. Cell $2003 ; 115$ : 787-98.

54. Grewal SI, Moazed, D. Heterochromatin and epigenetic control of gene expression. Science 2003; 301: 798-802.

55. Cimmino A, Calin GA, Fabbri M, et al. miR-15 and miR-16 induce apoptosis by targeting BCL2. Proc Natl Acad Sci USA $2005 ; 102$ : 13944-9.

56. Calin GA, Dumitru CD, Shimizu M, et al. Frequent deletions and downregulation of micro- RNA genes miR15 and miRl6 at 13ql4 in chronic lymphocytic leukemia. Proc Natl Acad Sci USA 2002 ; 99 : 15524-9.

57. Iorio MV, Ferracin M, Liu CG, et al. MicroRNA gene expression deregulation in human breast cancer. Cancer Res $2005 ; 65: 7065-70$.

58. Michael MZ, O' Connor SM, van Holst Pellekaan NG, et al. Reduced accumulation of specific microRNAs in colorectal neoplasia. Mol Cancer Res $2003 ; 1: 882-91$.

59. Chan JA, Krichevsky AM, Kosik KS. MicroRNA-21 is an antiapoptotic facto in human glioblastoma cells. Cancer Res $2005 ; 65: 6029$-33.

60. Johnson SM, Grosshans H, Shingara J, et al. RAS is regulated by the let-7 microRNA family. Cell $2005 ; 120: 635-47$.

61. Akao $Y$, Nakagawa $Y$, Naoe T. let-7 microRNA functions as a potential growth suppressor in human colon cancer cells. Biol Pharm Bull 2006 ; $29: 903-6$.

62. Takamizawa J, Konishi H, Yanagisawa K, et al. Reduced expression of the let-7 microRNAs in human lung cancers in association with shortened postoperative survival. Cancer Res 2004 ; 64 : 3753-6.

63. Lagos-Quintana M, Rauhut R, Yalcin A, et al. Identification of tissuespecific microRNAs from mouse. Curr Biol $2002 ; 12$ : 735-9.

64. Metzler M, Wilda M, Busch $K$, et al. High expression of precursor microRNA-155/BIC RNA in children with Burkitt lymphoma. Genes Chromosomes Cancer $2004 ; 39$ : 167-9.

65. Eis PS, Tam W, Sun L, et al. Accumulation of miR-155 and BIC RNA in human B cell lymphomas. Proc Natl Acad Sci USA 2005 ; 102 : 3627-32.

66. He L, Thomson JM, Hemann MT, et al. A microRNA polycistron as a potential human oncogene. Nature $2005 ; 435: 828-33$.

67. O’Donnell KA, Wentzel EA, Zeller KI, et al. c-Myc-regulated microRNAs modulate $\varepsilon 2 \mathrm{Fl}$ expression. Nature $2005 ; 435: 839-43$.

68. Hayashita $Y, 0$ sada $H$, Tatematsu $Y$, et al. A polycistronic microRNA cluster, miR-17-92, is overexpressed in human lung cancers and enhances cell proliferation. Cancer Res 2005 ; 65 : 9628-32.

69. Bandres $\varepsilon$, Cubedo $\varepsilon$, Agirre X, et al. Identification by Real-time PCR of 13 mature microRNAs differentially expressed in colorectal cancer and non-tumoral tissues. Mol Cancer $2006 ; 5: 29$.
TIRÉS À PART

દ. Ogier-Denis 